\title{
XVII. On the electric origin of the heat of combustion
}

\section{J.P. Joule Esq.}

To cite this article: J.P. Joule Esq. (1842) XVII. On the electric origin of the heat of combustion , Philosophical Magazine Series 3, 20:129, 98-113, DOI: 10.1080/14786444208650532

To link to this article: http://dx.doi.org/10.1080/14786444208650532

曲 Published online: 01 Jun 2009.

Submit your article to this journal $\llbracket$

Џ Article views: 6

Q View related articles ¿ 
Arabians as they took theirs from Ptolemy. The 7800 miles which he assumed for the diameter of the earth might indeed be taken as well from the reputed result of the Arabians as from his own measure; and having assumed this, he could dispense with all the rest. But in whatever way he camẹ by his result, its near approximation to the true value of the degree must be considered as a very remarkable circumstance. It is singular that the historian of his life (Plantius), who was his intimate friend, does not once allude to the measure of the earth, although it was a feat in which, as Lalande remarks, Fernel might reasonably be expected to have prided himself*.

Fernel was court physician to Henry II. of France. In his early years he cultivated mathematical studies, but abandoned them upon his marriage and devoted himself exclusively to the practice of his profession, by which he acquired a large fortune. He died in 1558, at the age of seventy-two. A gossiping account of him may be seen in Bayle's Dictionary. I am, Gentlemen, yours faithfully,

Serjeants' Inn, Jan. 6, 1842. T. Galloway.

XVII. On the Electric Origin of the Heat of Combustion. By J. P. Joule, Esq †.

[Illustrated by Plate 11I.]

1. $\mathrm{N}$ the papers which $\mathrm{I}$ had some time ago the honour of communicating to the Royal Society, I related an investigation concerning the calorific effects of voltaic electricity, and stated my opinion with regard to the heat evolved by combustion and certain other chemical phænomena. In the present paper $I$ intend to bring forward some experiments in confirmation of my theory, and to prove that the heat of combustion, terminating in the formation of an electrolyte, is the consequence of resistance to electric conduction.

2. We have seen that when those chemical actions which are not the sources of transmitted electricity are allowed for, the heat evolved from any part of the voltaic apparatus is the effect of the resistance which is presented by that part to the electric current; and that hence it necessarily follows, that the total voltaic heat generated by the action of any closed galvanic pair is proportional to the number of chemical equivalents which have been consumed in the act of propelling the current, and the intensity of the galvanic arrangement. Now, if it can be shown that the quantity of heat which is evolved by ordinary

[* On the subject of this paper see an additional communication from Professor de Morgan, at p. 116.-EDir.]

t Read before the Literary and Philosophical Society of Manchester, November 2, 1841; and now communicated by the Author. 
Phul Mag. S. 3. Vol: XX. Plate III.

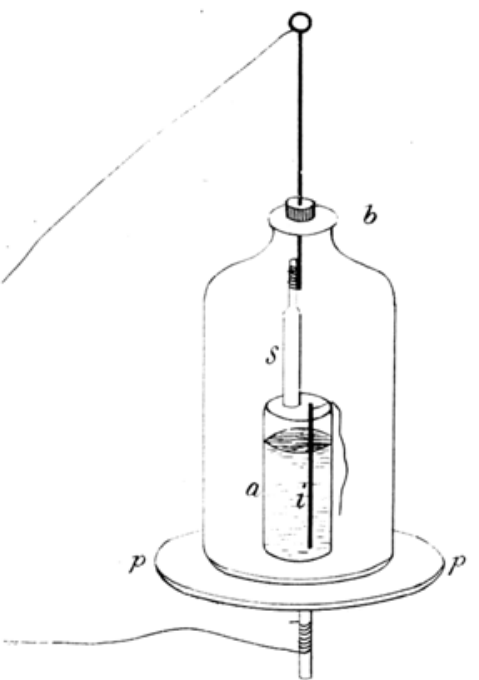

Fig: 1 .

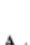

Fig. 2.
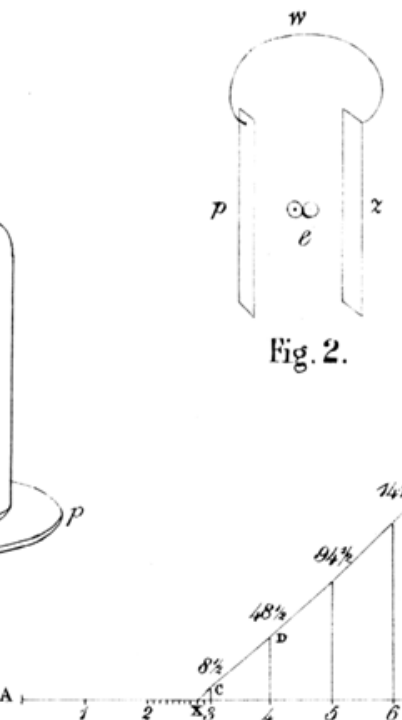

Fiǵ: 4 .

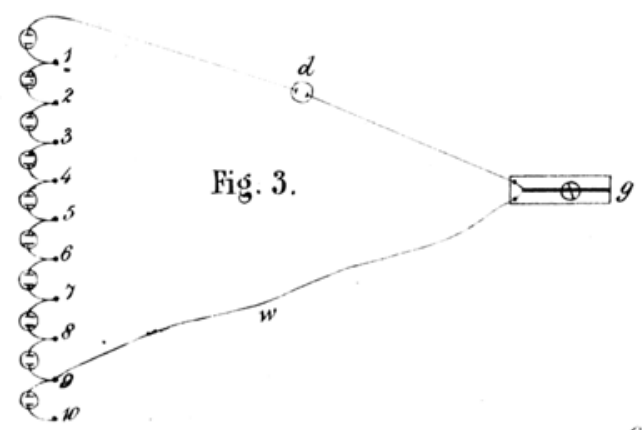

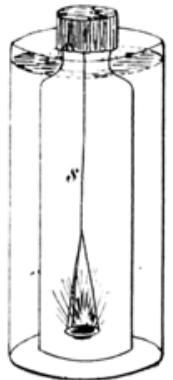

Fig: 7.

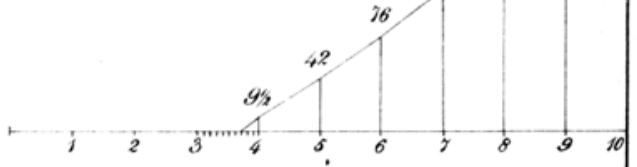

Fig: 5 .

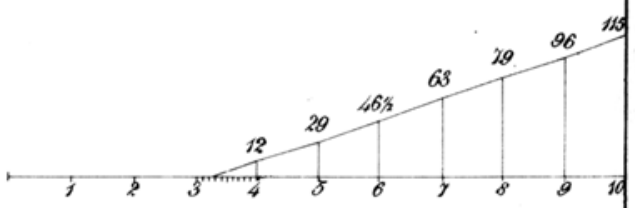

Fiğ: 6 . 
chemical combination is the same as calculation founded on these facts would lead us to expect, no reasonable doubt can be entertained that it also is the product of resistance to electric conduction.

3. In studying the character of the heat of combustion, the first point was to determine the intensities of the affinities of different combustibles for oxygen. For this purpose I have, in accordance with the views which were first stated by Davy, and bave since been adopted by the most eminent electricians, made use of the measure of these intensities which is afforded by the electric current.

4. I had not proceeded far before some curious phænomena were observed, which, though not very well understood, have long been known* to electricians. I shall notice these first, because of their important bearing upon subsequent reasonings and conclusions.

5. I was working with an arrangement consisting of iron, platinized silver, and dilute sulphuric acid. The circuit was closed by a galvanometer, the coil of which consisted of 119 turns of thin-silked copper wire, forming a rectangle, measuring 1 foot by 6 inches. The needle indicated a pretty constant deviation of $20^{\circ}$, but on moving the platinized silver backwards and forwards the needle advanced gradually to $4.0^{\circ}$, where it was kept for some time by continuing the agitation. As soon as the motion of the platinized silver was discontinued, the needle resumed its former position. Similar effects were produced by stirring the liquid, and thus causing it to impinge against the platinized silver.

6. I repeated the above experiment many times with similar results, but $I$ found that whenever a large quantity of hydrogen had been evolved from the liquid by the action of the pair, or otherwise, the phænomena were not well produced. This circumstance convinced me that the effects were due to atmospheric air held in solution by the liquid, and that the displacement of a part of it by the hydrogen had occasioned their partial prevention. My opinion was confirmed by the following experiment.

7. I filled three quarters of the contents of a glass flask with dilute sulphuric acid, and then placed it over the flame of a spirit-lamp until I judged that all the atmospheric air had been

* In 1830, Mr. Sturgeon remarked that when two pieces of iron are placed in dilute muriatic acid, the agitation of one of them will make it operate as copper in the copper-zine battery: also, that if two pieces of iron are immersed in succession in a solution of nitrous acid, the iron last im. mersed will act as copper in the copper-zinc battery.-Recent Experimental Researches, p. 46-49. We shall hereafter see the true cause of these phæ. nomena. 
boiled out. I then removed the lamp, and immediately placed in the mouth of the flask a cork, through which a small piece of platinized silver and a stout iron wire had been passed. On connecting the metals with the galvomometer (5.) its needle was deflected to $32 \frac{1}{2}^{\circ}$, and on shaking the flask very briskly it could not be made to advance further than to $34^{\circ}$. This advance, slight as it is, was probably entirely occasioned by air, which, notwithstanding my precautions, had found its way into the upper part of the flask.

8. The phænomena originated entirely from the platinized silver; and although a slight advance of the needle was sometimes produced by agitating the iron, it was not difficult to see that the real cause was the propulsion thereby occasioned of the aërated liquid against the negative* element, for when this was avoided no advance of the needle could be produced by agitating the positive metal.

9. I thought it probable that an increase of the intensity of the current would be produced by directing a stream of oxygen gas against the negative element. On making the experiment, I found that the needle advanced a few degrees, and that the same effect could be produced by a stream of hy. drogen. There could be no doubt that the increase of intensity arose rather from the agitation of the liquid than from any specific action of the gases, and that this experiment was essentially the same as that described in (5.).

10. I impregnated some dilute sulphuric acid with a very small quantity of oxygen, according to Thenard's process, and then immersed into it a plate of platinized silver and a rod of iron, both properly communicated with the galvanometer. The needle stood for the first few seconds at $68^{\circ}$; in three minutes it declined to $50^{\circ}$; in five minutes more to $49^{\circ}$, and in another five minutes to $48 \frac{1}{2}^{\circ}$. On agitating the platinized silver so as to bring it repeatedly in contact with the yet undecomposed deutoxide of hydrogen, the needle advanced to above $60^{\circ}$. The same pair, immersed in common acid, would have deflected the needle no further than $29^{\circ}$ or $30^{\circ}$.

11. The effect of the presence of oxygen at the negative element is well observed by making it, in water, the positive electrode of a voltaic battery. By this means oxygen is deposited on its surface, and is there ready to produce an extraordinary intensity. This deposit of oxygen is in fact the cause of the action of Ritter's secondary piles.

12. The following was also a very convenient method of

* To avoid misconception, it is perhaps as well to observe that I call those elements of the voltaic battery negative, which attract or combine with those bodies which are called "positively electrical," or "cations." 
showing the increase of intensity arising from the presence of oxygen. Some dilute sulphuric acid was agitated with chlorine until the former had taken up as much of the gas as it could. By pouring a solution of sulphate of oxide of silver into the liquid, I now precipitated chloride of silver, leaving sulphuric acid and oxygen in solution. When a pair, consisting of platinized silver and iron, was placed in the acid thus prepared, the galvanometer was permanently deflected $50^{\circ}$, and by agitating the platinized silver the needle advanced as far as $60^{\circ}$. When a piece of amalgamated zinc was substituted for the iron the permanent deflection of the needle was $65^{\circ}$, and by agitating the negative element as before, the needle advanced to $70^{\circ}$. Had the same pairs been immersed in a simple solution of sulphuric acid, the permanent deflections would have been no greater than $30^{\circ}$ and $63^{\circ}$.

13. Similar results were obtained with the solution of chlorine, as might have been anticipated from its strong affinity for hydrogen.

14. From the above experiments, we see that the agitation of the negative element is productive of an increase of intensity, simply because it is thereby brought into contact with bodies capable of combining with the hydrogen, which would otherwise have been evolved from it. When those bodies are present in considerable quantities, as in (10.), (12.) and (13.), the intensity of the current is great, even though the pair be left quiet, because then the negative plate can collect them readily upon its surface. Again, by causing the current to encounter great resistance, the effects of agitation which we have noticed are proportionally increased, because then the number of particles required for neutralizing the hydrogen is less. Hence it is that when $I$ have used a resistance of 500 or 600 yards of thin wire, I have frequently found the deviation of the needle (even when the pair was left quiet in a common solution of sulphuric acid) considerably greater than was due to that resistance. This is also the probable reason why De la Rive in one instance* found the intensity of the copper-zinc pile the same, whether charged with water or nitric acid.

15. In the course of the preceding experiments I was forcibly struck with the very great intensity of the pairs at the moment of their immersion, compared with that which they were able to maintain permanently. It appeared to me that the theories which had been put forth to explain the first effect of immersion, though seemingly plausible with regard to the zinc battery, were not at all equal to account for the same phæno-

* Ann. de Chimie, 1836, part i. p. 179. 
menon as existing to a far greater extent when iron is used as a positive element.

16. A rod of iron and a small plate of platinized silver were immersed in a dilute solution of sulphuric acid. On connecting them with the galvanometer (5.), the needle was permanently deflected $29 \frac{1}{2}^{\circ}$. After a few preliminary trials to ascertain the proper point, I caused the needle to be maintained by a glass weight at $55^{\circ}$, beyond which it was free to travel. I then exposed the platinized silver to the air during one minute of time. On re-immersing it the needle sprang as far as $60^{\circ}$, and then immediately recoiled to its resting place at $55^{\circ}$, thus indicating a transitory current of about $57 \frac{10}{2}$.

17. On exposing the platinized silver for $5^{\prime \prime}$ only, the transitory current, ascertained in a manner similar to that just mentioned, was $41^{\circ}$.

18. Greater effects were obtained by washing and drying the platinized silver before it was immersed. In this way the needle, adjusted at $62^{\circ}$, would spring as far as $66^{\circ}$, indicating a transitory current of about $64^{\circ}$. Having now removed the glass weight, the needle took up a permanent position at $29 \frac{1}{2}^{\circ}$, as at the beginning of the experiments.

19. When, instead of the platinized silver, the positive element (iron) was exposed to the air, whether simply or in conjunction with washing and drying, no appreciable increase of intensity was occasioned by its immersion. And although, on the repetition of the experiment, I sometimes observed slight effects, I conceive that they were owing to the power which the negative element seems to possess of collecting upon its surface the air held in solution by the circumambient liquid.

20. With an arrangement of platinized silver and amalgamated zinc, I obtained results of a similar though less striking character. The galvanometer indicated a permanent deflection of $62^{\circ}$, and after washing and drying the platinized silver, I had a transitory deviation of $72^{\circ}$. The immersion of the amal. gamated zinc, after washing and drying, produced no effect.

21. The maximum effects of immersion were produced in the following manner. A plate of silver was rubbed with a little nitric acid, and then exposed to a red heat, by means of which the film of nitrate of oxide of silver was decomposed and metallic silver reduced*. When the plate prepared in

* By this process all the oxygen is not driven off, but a considerable quantity remains adhering to the silver so tenaciously that it is not entirely removed by making the plate quite bright with glass paper. The oxygen thus deposited (it can hardly, I think, be considered as chemically combined with the silver) is the cause of the great intensity of the current immediately after immersion. By simply heating the silver to rerlness the same general effects can be produced, though not to the same extent 
this way was associated in dilute sulphuric acid with a piece of iron, the needle would deviate $63 \frac{1}{2}^{\circ}$ for some time, and then gradually decline until it took up a permanent situation at $29 \frac{1}{2}^{\circ}$. By experimenting in the same way with amalgamated zinc as a positive element, I had a transitory deflection of $76^{\circ}$, and a permanent deflection of $63^{\circ}$.

22. Very trifling transitory effects were obtained by the immersion of iron, when that metal was associated with amalgamated zinc. But this might have been anticipated, because the transitory current is owing to the presence of oxygen on the negative plate, and it is obvious that the hydrogen evolved by the local action of the iron, would, whilst in a nascent state, combine with that oxygen, and thus prevent a great part of it from exercising any influence upon the intensity of the current.

23. An experiment was also made with an arrangement of copper, amalgamated zinc, and dilute sulphuric acid. It was able to deflect the needle $51^{\circ}$ pretty permanently. On washing and drying the copper, and experimenting as in (16.), I observed a transitory deflection of $72^{\circ}$. This experiment deserves attention, because it shows that the transitory current occasioned by the copper is the same as that exhibited by platinized silver when experimented with in the same way (20.). I take it as an argument, that when copper is in its best state it forms with amalgamated zinc a battery as intense as the platinized silver.

24. That the transitory currents which we are discussing are not occasioned by the diffusion of the salt formed about the positive element during the cessation of voltaic action, is obvious from the fact that (when the proper precautions are observed) they are not produced by the agitation (8.), or by the immersion (19. and 20.) of the metal about which the salt is formed. And if anything can render this more evident, it is the fact that the immersion of the copper plate of a Daniell's battery causes the needle to advance little or no higher than its permanent situation, as might have been anticipated from the theory which refers the transitory effects to chemical combination at the negative plate, on account of the slight affinity of copper for oxygen. The following experiments are also decisive of this question.

25. A glass jar, $a$, fig. 1 , containing some dilute sulphuric acid, was placed upon the plate $p p$ of an air-pump. A small rod of iron, $i$, was immersed in the liquid, and connected by means of the pump-plate to the galvanometer (5.). An open receiver, $r$, was now placed over the jar, and the ground brass plate $b$, with its stuffing-box and sliding rod (the latter having the small piece of platinized silver, $s$, affixed to its extremity), 
was placed on the top of the receiver. A copper wire, fastened to the ring of the sliding rod, connected the platinized silver with the galvanometer.

26. The sliding rod was now moved until the platinized silver in connexion with it was immersed in the acidulated water. Then the pump was worked until a very excellent vacuum was obtained, and so tight was every part of the apparatus that it could be left alone for half an hour without the admission of any appreciable quantity of air. The galvanometer indicated a permanent deflection* of $27^{\circ}$. I now placed a piece of glass so as to prevent the needle from going lower than $27^{\circ}$, and by means of the sliding rod I removed the platinized silver entirely out of the acid. After it had been exposed during a quarter of an hour I re-immersed it, when the needle sprang from $27^{\circ}$ to $30^{\circ}$ and back, indicating a transitory deflection of about $28 \frac{1}{2}^{\circ}$. Although the effect of immersion exhibited by this experiment is extremely small, it appeared to be almost entirely occasioned by the repose of the electric condition of the iron, for when, instead of entirely withdrawing the platinized silver, its extremity was just allowed to tonch the liquid, the transitory deffection was only $27 \frac{1}{2}^{\circ}$ after an exposure during a quarter of an hour.

27. On admitting a quantity of air into the receiver sufficient to counterbalance the pressure of one inch of mercury, the effects of immersion were considerable after a very short exposure of the platinized silver. In a quarter of an hour it collected upon its surface sufficient oxygen to cause the needle to spring from $27^{\circ}$ to $78^{\circ}$, whether it had or had not remained in contact with the liquid during its exposure.

28. When, instead of the vacuum, I used an atmosphere of hydrogen, the exposure of the platinized silver for any length of time did not render the current more intense at the moment of immersion than it remained permanently. And even when the hydrogen was diluted with one quarter of its bulk of atmospheric air the transitory effects did not appear, on account, no doubt, of the union $\uparrow$ of the oxygen with the hydrogen as fast as the former, or both, collected upon the plate. On using a mix ture of equal bulks of hydrogen and air, the transitory effects were very small, even after the platinized silver had been exposed for ten minutes.

29. I made several experiments with carbonic acid, but the

* No change in the permanent deflection of the needle was occasioned by the removal of atmospheric pressure.

t The phænomenon of Dcebereiner, so fully investigated by Faraday, to whose paper, published in the Phil. Trans. for $\mathrm{J} 834$, I refer the reader for some valuable observations on the power possessed by metals of ccndensing gases upon their surfaces. 
transitory currents did not entirely disappear as was anticipated. The gas, though prepared carefully and in different ways, could not be obtained perfectly pure, and when exposed to an alkaline solution, $\frac{1}{2} 0$ th of it would remain uncondensed. In order therefore to remove any free oxygen which the gas might contain, I exposed it during two days and two nights to the action of a stick of phosphorus. After this, immersion caused no, or at most, very trivial transitory effects ; but on admitting only one per cent. of oxygen they became very considerable,-a striking example of the power possessed by metals of collecting and condensing oxygen upon their surfaces. I do not bring forward this experiment as a proof of the entire non-action of carbonic acid, because the phosphorus was found to have decomposed it partially.

30. All these phænomena are easily understood, if, with the great body of philosophers, we keep in view the intimate relation which subsists between chemical affinity and the electric current. For let $p$, fig. 2, represent a plate of platinum; $z$, a plate of zinc, or other electro-positive metal; and $c$, one of a series of atoms of water extending from $p$ to $z$. The intensity of the current along the wire $w$, is proportional to the affinity of oxygen for the positive metal, minus the affinity of oxygen for hydrogen. But if $p$ be covered with a film of oxygen, the current will be entirely proportional to the affinity of the positive metal for oxygen. In the former case, $c=z-h$; in the latter, $c^{\prime}=z$.

31. Considering these equations, it is obvious why, as I have observed (15.), the transitory currents are better exhibited with iron than with zinc as a positive element; for in proportion to the smallness of $z$, provided it remain greater than $h$, will the difference between $c$ and $c^{d}$ be more manifest. If $c^{l}-c$ be the same for both iron and zinc, we shall have a proof of the accuracy of these principles.

32. Thus from (21.), turning the deflections of the needle into quantities of electricity, we have $63 \frac{1}{2}^{\circ}=0^{\circ} .034 \mathrm{Q}$, and $29 \frac{1}{2}^{\circ}=0^{\circ} .0072 \mathrm{Q}$, of which the difference is $0^{\circ} .0268 \mathrm{Q}$, when iron is the positive element. We have also $76^{\circ}=0^{\circ} .056 \mathrm{Q}$, and $63^{\circ}=0^{\circ} .027 \mathrm{Q}$, of which the difference is $0^{\circ} 029 \mathrm{Q}$, when zinc is the positive element. I consider these differences as nearly equal as could have been expected from the nature of the experiments.

33. I might now proceed to consider in detail several phænomena (such as the very rapid corrosion of metals when they are exposed to the joint action of air and moisture, \&c.) which are occasioned by the great intensity of galvanic action in consequence of the mixture of oxygen with the liquid But I hasten to fulfil my principal design. 
Intensities of the Affinities which unite bodies with Oxygen.

34. In order to ascertain the intensities of galvanic arrangements, we may either use a galvanometer furnished with a short and thick wire, or with a long and thin wire (within certain limits (14.)). In the former case the calculations must be conducted on the principles of $\mathrm{Ohm}$; in the latter it is only necessary to take care that the resistances of the pairs under comparison are pretty nearly equal, in order that the deviations of the needle may be depended upon in calculating the intensity of the current. I have adopted the latter plan on account of the superior facilities which it presents.

35. Affinity of zinc for oxygen.-From (32.) we have the intensity of the action of zinc $=0^{\circ} \cdot 056 \mathrm{Q}$; and the intensity required for the electrolysis of water $=0^{\circ} .029 \mathrm{Q}$. Hence $29: 56:: 1$, the affinity of hydrogen, $: 1 \cdot 93$, that of zinc for oxygen.

36. Affinity of iron for oxygen, likewise obtained from(32.), is $1 \cdot 27$, for $268: 340:: 1: 1 \cdot 27$.

37. Affinity of potassium for oxygen.-Twenty grains of potassium were combined with about ten ounces of mercury. The amalgam was poured into a wooden cup, into the bottom of which a copper wire connected with the galvanometer (5.) had been let. At about half an inch above the surface of the amalgam I secured a piece of platinum, also in connexion with the galvanometer. On pouring dilute sulphuric acid into the cup the needle was deflected $74^{\circ}\left(=0^{\circ} .05 \mathrm{Q}\right)$ during three successive minutes, but the local action of the amalgam was so vigorous that at the end of this interval of time most of the potassium was dissolved, and the needle declined very fast. On treating 20 grains of zinc in precisely the same manner, I had a deviation of $49^{\circ}\left(=0^{\circ} 0152 \mathrm{Q}\right)$. Hence

and

$$
\begin{aligned}
& k-h=0.05, \\
& z-h=0.0152,
\end{aligned}
$$

whence

But from (35.), $z=1 \cdot 93$, and $h=1$; therefore $k$, the affinity of po assium, $=4.06$.

38 . It is necessary ${ }_{2}$ however, to pay attention to the circumstances under which the experiments were made, in order to obtain correct ideas concerning the above intensities of affinity. The increase of the intensity of the voltaic apparatus by heat is by no means great; and as all the experiments were conducted at conmmon temperatures, no regard need be paid to $i t$. But then the intensities of affinity were obtained by comparing currents which had been produced under peculiar circumstances with regard to the cordition of the elements of the galvanic arrangements: in one case the hydrogen was evolved in a 
gaseous state; whilst in the other, the hydrogen, by combining with free and condensed oxygen, did not escape. Now we shall see from the following experiments that electric intensity is expended in the act of converting a body into the gaseous state.

39. I took ten glass jars (see fig. 3), made them perfectly clean and dry *, and placed them in series on a non-conducting substance. Into these I poured a quantity of dilute sulphuric acid, taking care not to wet the glass within an inch of the top of each. Pairs of platinized silver and amalgamated zinc were placed in the jars, and connexions, furnished with the mercury cups $1,2,3, \& c$. , were established between them seriatim. A decomposing cell, $d$, furnished with platinum wires, was connected on one hand with the battery, and on the other with the galvanometer (5.). Lastly, I provided a copper wire, w, by means of which connexion could be conveniently made between the galvanometer $g$, and any of the mercury cups, 1 , 2, 3, \&c.

40 Into $d$ I poured a small quantity of dilute sulphuric acid. Then, by placing the wire $w$ in each of the mercury cups, beginning at 10 and ending at 10 , I observed the deviations of the galvanometer contained in the following table.

\begin{tabular}{|c|c|c|c|c|c|}
\hline \multirow{2}{*}{$\begin{array}{l}\text { No. of pairs } \\
\text { in action. }\end{array}$} & \multicolumn{4}{|c|}{ Deflections of Galvanometer. } & \multirow{2}{*}{$\begin{array}{l}\text { Comparative quantities } \\
\text { of Electricity. }\end{array}$} \\
\hline & Down. & Up. & & & \\
\hline 10 & $72 \frac{1}{2}$ & 71 & $7 i$ & 45 & 341 \\
\hline 9 & $69^{2}$ & 68 & 68 & 30 & 284 \\
\hline 8 & $65 \frac{1}{4}$ & 65 & 65 & 7 & 236 \\
\hline 7 & $60^{4}$ & 60 & 60 & 0 & 188 \\
\hline 6 & 53 & $53 \frac{2}{3}$ & 53 & 20 & 141 \\
\hline 5 & 42 & $43^{3}$ & 42 & 30 & $94 \frac{1}{2}$ \\
\hline 4 & $25 \frac{1}{2}$ & $27 \frac{3}{4}$ & 26 & 37 & $48 \frac{2}{2}$ \\
\hline 3 & $5 \frac{2}{2}$ & $5 \frac{4}{2}$ & 5 & 30 & $8 \frac{1}{2}$ \\
\hline 2 & $0^{2}$ & $0^{2}$ & 0 & 0 & $0^{2}$ \\
\hline 1 & 0 & 0 & 0 & 0 & 0 \\
\hline
\end{tabular}

41. Now if we divide the straight line, A B, fig. 4, into ten equal parts, representing pairs on Mr. Smee's plan, and if at each division we erect straight lines, perpendicular to A B and proportional to the comparative quantities of electricity just given, the principles of electric action demand that the line drawn through the extremities of those perpendiculars

* It is necessary to be very careful in insulating the apparatus, in order to obtain the maximum intensity of a battery. The divided porcelain trough has frequently great conducting powers (particularly when the glaze has been partially destroyed), which render it unfit for accurate experiments. 
should be straight. It is in fact so nearly a straight line, that its slight discrepancies therefrom may be properly referred to unavoidable errors of experiment. Produce the straight line $C D$ so as to meet $A B$ in $X$, and the straight line $A X$, equal to $2 \cdot 8$, will indicate the number of pairs necessary to decompose water.

42. Fig 5 represents an experiment of the same kind, with a solution of sulphate of oxide of zinc in the decomposing cell. Oxide of zinc was decomposed, the oxygen being evolved at the positive, and the zinc being reduced at the negative electrode. The intensity necessary to decompose oxide of zinc is equal to that of 3.7 of Mr. Smee's pairs.

43. With sulphate of protoxide of iron I did not at first succeed, on account of the formation of peroxide at the positive electrode. However, by placing the negative electrode among some crystals of the salt, pouring water thereon, and suspending the positive electrode in the water, I obviated that difficulty, and obtained the results which are projected in fig. 6, and which indicate 3.3 pairs as the intensity necessary to decompose protoxide of iron.

44. Now from (41.) and (42.) we have (using the same letters as before) 2.8 pairs $=h$, and 3.7 pairs $=z$; whence $2.8 z=3.7 h$, and $z=1.32 h$; or, in other words, the intensity required to separate oxide of zinc into metal and gas is to the intensity required to separate water into its gaseous elements as $1.32: 1$. But from (35.), the intensity produced by the union of non-gaseous oxygen with zinc is to the intensity necessary to separate water into non-gaseous oxygen and gaseous hydrogen as $1.93: 1$; and $1.32: 1:: 1 \cdot 93+1.9: 1+1 \cdot 9$. Wherefore, the intensity necessary to give oxygen the gaseous form is to the intensity necessary to separate water into nongaseous oxygen and gaseous hydrogen as $1.9: 1$.

4.5. Thus we see that a very great intensity of current is employed in changing the condition of bodies, as well as in separating them from their combinations. The field of investigation here opened is very extensive, but I may not at present enter further upon it. I will only remark, that if the intensity necessary to convert a body into a different state, compared with the heat or cold due to the mechanical or other production of that different state, be such as accords with the relations of intensity and heat which we observe in the voltaic apparatus, we have a proof that some of the effects which are usually referred to "latent heat," are in fact nothing more than the recondite operations of resistance to electric current*.

46. In our investigation into the cause of the heat of com-

* Some experinents, which I have not time to refer to at present, render this hypothesis more than probable. 
bustion, it will be necessary to deduce our calculations from the electric intensity which is required in order to reduce the product of combustion to the state in which its elements were prior to combustion. The following is a list of these intensities, reckoning the decomposition of water into its gaseous elements as unity.

47. Intensity necessary to decompose oxide of zinc into gaseous oxygen and metal, from (42.) and (44.), is 3.7 pairs of Smee's battery, or $1 \cdot 32 h$.

48. Intensity necessary to decompose protoxide of iron into gaseous oxygen and metal.-From (43.), 3.3 of Smee's pairs $=i ;$ and from (41.), 2.8 pairs $=h$; whence $2.8 i=3.3 h$, or $i=1 \cdot 18$.

49. Intensity necessary to decompose potassa into potassium and gaseous oxygen.-From (44.) and (37.) we have 1.93 $+1 \cdot 9$ $: 4.06+1.9:: 1.32 h: 2.05 h$, the intensity required, which may be otherwise expressed by $5^{\circ} 74$ of Smee's pairs.

\section{Heat evolved by Combustion, when it terminates in the forma- tion of an Electrolyte.}

50. Finding that our information on the quantity of heat evolved by the combustion of metals was not very satisfactory, I have, without wishing to depreciate the labours of Dulong, Despretz and others, thought it right to bring forward such of my own experiments as are necessary in order to make my investigation complete.

51. I provided two glass jars. The smaller had an internal capacity of 90 cubic inches; and when placed within the other jar, as represented by fig. 7, the space left between the two was sufficient to contain three pounds of water. By means of a scale, $s$, suspended by wire from a thick fold of moistened paper, I was able to introduce a combustible within an atmosphere of oxygen, and by means of a heavy weight $I$ could keep the paper sufficiently close to the top of the jar to prevent the escape of any considerable quantity of heated air, while at the same time it was not so tight as to prevent the admission of air as the oxygen was consumed. The increase of the temperature of the water was measured by a thermometer of great sensibility.

52. The heat evolved by the combustion of zinc was ascertained in the following manner. The smaller jar was filled with oxygen, placed in the other jar, and surrounded by three pounds of water, the heat of which was contrived to be as much below the temperature of the surrounding air as it was expected to exceed it at the close of the experiment. A piece 
of phosphorus, weighing 0.4 grain, was then put into the scale, and over it I placed a heap of fine zinc turnings, weighing 50 grains. I now ignited the phosphorus, and plunged the scale into the inner jar. After the combustion had terminated, and the heat thereby evolved had been evenly distributed throughout the water by stirring, the increase of temperature was noted. The contents of the scale were then thrown into dilute sulphuric acid, and the volume of hydrogen thereby evolved indicated the quantity (generally about 15 grains) which had not been burnt. 'Two-tenths of a degree of heat were deducted from the observed heat, on account of the phosphorus, and an allowance having been made, on account of the capacity of glass for heat, the results were reduced to the standard of one pound of water.

53. The mean of several experiments conducted in the above manner, showed that the heat evolved by the combustion of $32 \cdot 3$ grains of zinc is able to increase the temperature of a pound of water by $10^{\circ} \cdot 8$.

54. The heat evolved by the combustion of iron was ascertained in a similar way. The iron was in the state of fine wire, and that portion of it that was not burnt was carefully collected, weighed, and deducted from the original quantity. The mean of several trials indicated that 28 grains could increase the temperature of a pound of water by $9^{\circ} .48$.

55. Heal evolved by the combustion of potassium.-This metal, in pretty large lumps, was introduced into an atmosphere composed of equal bulks of oxygen and air. I then introduced a stout iron wire, sharpened at the end, into the jar, and with it I cut the potassium into small pieces. Under this treatment it soon became so soft, that every time the rod was lifted it would draw out a string of metal. In this state it often ignited, and the experiment was spoiled on account of the partial formation of peroxide. However, by careful management, I succeeded in making some good experiments, in which nearly all the potassium was converted into potassa; and the exact quantity of unoxidized metal was ascertained by observing the volume of hydrogen evolved when the contents of the scale were exposed to the action of water. The mean of these showed that the heat evolved by the conversion of 40 grains of potassium into potassa is able to increase the temperature of a pound of water by $17^{\circ} \cdot 6$.

56. Heat evolved by the combustion of hydrogen.-The gas was burned in an armosphere of oxygen, diluted with common air, by means of a jet furnished with a very narrow bore. A grain of hydrogen evolved as much heat as is able to increase the temperature of a pound of water by $8^{\circ} .36$. 
57. We shall now proceed to examine how far the theory of resistance to electric conduction agrees with the above experimental results.

58. We have seen (47.), (48.) and (49.) that the intensities of the affinities which unite gaseous oxygen with zinc, iron, potassium and gaseous hydrogen, are as $1.32,1 \cdot 18,2 \cdot 05$ and 1 ; and the proportional quantities of heat which were generated by the combustion of the equivalents of these bodies are $10^{\circ} \cdot 8$, $9^{\circ} \cdot 48,17^{\circ} \cdot 6$, and $8^{\circ} \cdot 36$, or $1 \cdot 29,1 \cdot 13,2 \cdot 105$ and 1 , a ratio which is very nearly the same as that of the intensities just given. Hence we see that the quantities of heat which are evolved by the combustion of the equivalents of bodies are proportional to the intensities of their affinities for oxygen. Now I proved in my former paper * that a similar law obtains in the voltaic apparatus, in consequence of its heat being produced by resistance to conduction. And hence we have an argument that the heat of combustion has the same origin.

59. But our proof of the real character of the heat of combustion is rendered more complete by regarding quantities as well as ratios of heat. From the quantity of heat generated by the motion of a given current along a wire of known resistance, we can deduce the quantities of heat which, according to the theory of resistance to electric conduction, ought to be produced by the combustion of bodies; and then these theoretical deductions may be compared with the results of experiment.

60. The mean of three careful experiments detailed in my former papert, shows that if a wire, the resistance of which is an unit, be traversed by an electric current of $1^{\circ .88} \mathrm{Q} \ddagger$ for one hour, the heat evolved by that wire will be able to increase the temperature of a pound of water by $15^{\circ} \cdot 12$. Now I have ascertained experimentally that a pair consisting of analgamated zinc and platinized silver, excited by dilute sulphuric acid, is able to propel a current of $0^{\circ} \cdot 168 \mathrm{Q}$ against the whole resistance of the circuit, when that resistance is $5 \cdot 2$; consequently, a similar pair can propel a current of $0^{\circ} .168 \mathrm{Q} \times 5.2$ $=0^{\circ} .874 \mathrm{Q}$ against the resistance which $\mathrm{I}$ have called an unit. But from (42.), the intensity necessary to separate oxide of zinc into zinc and gaseous oxygen is to the intensity of one of Smee's pairs as $3 \cdot 7: 1$. Consequently, the electricity produced

* Philosophical Magazine, October 1841, S. 3. vol. xix. p. 275. (70.)

$\uparrow$ Ibid. p. 266.

$¥ I$ beg to remind the reader that my degree, expressed thus $\left(l^{\circ} Q\right)$, indicates that quantity of current electricity which, after passing constantly during one hour, is found to have electrolized a chemical equivalent expressed in grains; as, 9 grains of water, 36 grains of protoxide of iron, $8 c$. 


\section{On the Electric Origin of the Heat of Combustion.}

by the union of zinc and gaseous oxygen must be sufficiently intense to propel a current of $0^{0.874} \mathrm{Q} \times 3.7=3^{\circ .234} \mathbf{Q}$ against an unit of resistance. Now $1^{\circ} .88 \mathrm{Q}$, when urged against an unit of resistance, was able in one hour of time to increase the temperature of a pound of water by $15^{\circ} .12$; therefore $3^{\circ} \cdot 234 \mathrm{Q}$ could, in the same circumstances, produce $\left(\frac{3.234}{1.88}\right)^{2}$ $\times 15^{0.12}=44^{\circ} \cdot 74$ of heat. But in (70.) of my former paper, I proved that the same quantity of heat should always (according to the theory which refers the whole of the heating power of the voltaic apparatus to resistance to the electric current) be produced by a given quantity and intensity of electrolysis, whether the resistance opposed to the current be small or great. Wherefore, the heat, which or these principles ought to be generated by the combustion of $3 \cdot 234$ equivalents of zinc, is $44^{\circ} .74$, or, in other words, one equivalent, or 32.3 grains of zinc, should generate heat sufficient to increase the temperature of a pound of water by $13^{\circ} .83$.

61 . Now, as 1 have before stated, the quantities of heat evolved by the combustion of the equivalents of bodies, ought, according to the theory of resistance to electric conduction, to be proportional to the intensities of their affinities for gaseous oxygen. These, in the cases of zinc, iron, potassium, and hydrogen, are $1.32,1 \cdot 18,2 \cdot 05$ and 1 . Hence $13^{\circ} \cdot 83,12^{\circ} \cdot 36$, $21^{\circ} \cdot 47$, and $10^{\circ} \cdot 47$, are the quantities of heat which ought, according to our theory, to be produced by the combustion of 32.3 grains of zinc, 28 grains of iron, 40 grains of potassium, and 1 grain of hydrogen.

62. By comparing these results of theory with the quantities of heat, $10^{\circ} 8,9^{\circ} \cdot 48,17^{\circ} \cdot 6$, and $8^{\circ} \cdot 36^{*}$, which were $(53-56$.) obtained from experiment, it will be seen that the former exceed the latter by about one quarter. Considering the difficulty of preventing some loss of heat, in consequence of the escape of air from the mouth of the inner jar (51.) during the first moments of combustion, \&c., it will, I think, be admitted that experiment agrees with the theory as well as could have been expected.

63. I conceive, therefore, that I have proved in a satisfactory manner that the heat of combustion (at least when it terminates in the formation of an electrolyte) is occasioned by resistance to the electricity which passes between oxygen and the combustible at the moment of their union. The amount of this resistance, as well as the manner of its opposition, is immaterial both in theory and in experiment; and if the resist-

* Crawford, whose method was well adapted to prevent loss of heat, obtained $9^{\circ} \cdot 6$. More recently, Dalton observed about $8^{\circ} \cdot 5$. 
ance to conduction be great, as it most probably is when potassium is slowly converted into potassa by the action of a mixture of oxygen and common air ; or little, as it probably is when a mixture of oxygen and hydrogen is exploded; still the quantity of heat evolved remains proportional to the number of equivalents which have been consumed, and the intensity of their affinity for gaseous oxygen.

64. That the heat evolved by other chemical actions, besides that which is called combustion, is caused by resistance to electric conduction, I have no doubt. I cannot, however, enter in the present paper upon the experimental proof of the fact.

Broom Hill, Pendlebury, near Manchester, October 5, 1841 .

XVIII. On the Development of the Cosines and Sines of Multiple Arcs. By Henry Malden, M.A., late Fillow of Trinity College, Cambridge, and Professor of Greek in University College, London *.

T the Philosophical Magazine for August 1841, [S. 3. vol. xix.] Mr. Booth has developed the cosine of a multiple arc in descending powers of the cosine of the ssimple arc, by the application of a new theorem in the calculus of finite differences, and has demonstrated that $\cos n \theta$ cannot be so expanded when $n$ is either negative or fractional.

As this is the case, it seems sufficient to present the expansion of the integral multiple in an easier way.

$$
\begin{aligned}
& \cos (n+1) \theta=2 \cos \theta \cdot \cos n \theta-\cos (n-1) \theta: \\
& \text { hence } \quad \frac{\cos n \theta}{\cos (n+1) \theta}=\frac{\cos n \theta}{2 \cos \theta \cdot \cos n \theta-\cos (n-1) \theta}, \\
&=\frac{1}{2 \cos \theta-\frac{\cos (n-1) \theta}{\cos n \theta}} ;
\end{aligned}
$$

and as the fractional term in the denominator is of the same form as the fraction on the first side of the equation, this may be developed in the form of a continued fraction, thus:

$$
\frac{\cos n \theta}{\cos (n+1) \theta}=\frac{1}{2 \cos \theta-\frac{1}{2 \cos \theta-\frac{1}{2 \cos \theta-\& c}}},
$$

in which $2 \cos \theta$ recurs $n$ times as the integral part of the partial denominators, and the last fractional part is

$$
\begin{aligned}
& \frac{\cos 0 \theta}{\cos \theta} \text {, or } \frac{1}{\cos \theta} \text {. } \\
& \text { * Communicated by the Author. }
\end{aligned}
$$

Phil. Mag. S. 3. Vol.20. No. 129. Feb. 1842. 